\title{
High Potential Columnar Nanocrystalline AlN Films Deposited by RF Reactive Magnetron Sputtering
}

Chengzhang $\operatorname{Han}^{1,2}$, Da Chen ${ }^{2, *}$, Yaozhong Zhang ${ }^{1}$, Dong $\mathrm{Xu}^{1}$, Yijian Liu ${ }^{1,2}$, Eric Siu-Wai Kong ${ }^{1}$, Yafei Zhang ${ }^{1, *}$

(Received 10 February 2012; accepted 12 March 2012; published online 19 March 2012)

\begin{abstract}
Columnar nanocrystalline aluminum nitride (cnc-AlN) thin films with (002) orientation and uniform texture have been deposited successfully on large silicon wafers by RF reactive magnetron sputtering. At the optimum sputtering parameters, the deposited cnc-AlN thin films show a c-axis preferred orientation with a crystallite size of about $28 \mathrm{~nm}$ and surface roughness (RMS) of about $1.29 \mathrm{~nm}$. The cnc-AlN thin films were well transparent with an optical band gap about $4.8 \mathrm{eV}$, and the residual compressive stress and the defect density in the film have been revealed by Ramon spectroscopy. Moreover, piezoelectric performances of the cnc-AlN thin films executed effectively in a film bulk acoustic resonator structure.
\end{abstract}

Keywords: Columnar film; Aluminum nitride; Piezoelectric effect; RF sputtering; Optical property

Citation: Chengzhang Han, Da Chen, Yaozhong Zhang, Dong Xu, Yijian Liu, Eric Siu-Wai Kong and Yafei Zhang, "High Potential Columnar Nanocrystalline AlN Films Deposited by RF Reactive Magnetron Sputtering", Nano-Micro Lett. 4 (1), 40-44 (2012). http://dx.doi.org/10.3786/nml.v4i1.p40-44

\section{Introduction}

AlN has been attracting considerable attentions because of its superior physical properties such as good thermal and chemical stability, high ultrasonic velocity, outstanding thermal conductivity, high electrical resistivity, excellent piezoelectric response, wide optical band gap, etc. Recently, there has been great interest in the excellent properties of AlN thin films for a wide variety of applications [1], such as optoelectronic devices, microelectronic devices and bulk acoustic wave devices [2]. Several methods, such as pulsed laser deposition (PLD), chemical vapor deposition (CVD), molecular beam epitaxy (MBE) and magnetron sputtering [3], have been reported to deposit AlN thin films. However, it is remain to be investigated for obtaining high quality highly oriented cnc-AlN thin films with expected properties, e.g. c-axis perpendicular to the substrate surface, high stiffness, low surface roughness, resistance to solvents, high conductive to heat, etc.

Reactive magnetron sputtering is considered to be a kind of potential deposition technique with advantages of being low temperature, low cost and controllable deposition conditions. Besides, the surface morphology, grain size and band gap energy of AlN films are connected with the deposition conditions [4]. A high degree of c-axis orientation and smooth surface of the AlN films are essential to the bulk acoustic wave devices, since the piezoelectric properties are strongly dependent on the crystallographic orientation of the film [5].

The aim of this work was to develop an advantageous synthesis condition of RF reactive magnetron sputter-

\footnotetext{
${ }^{1}$ Key Laboratory for Thin Film and Microfabrication of the Ministry of Education, Research Institute of Micro/Nano Science and Technology, Shanghai Jiao Tong University, Shanghai 200240, China

${ }^{2}$ Department of Applied Physics, College of Science, Shandong University of Science and Technology, 579 Qianwangang Road Economic \& Technical Development Zone, Qingdao Shandong Province, 266510, China

*Corresponding author. E-mail: yfzhang@sjtu.edu.cn, Tel: +86-021-3420-5665, Fax: +86-021-3420-5665

* Corresponding author. E-mail:phychenda@163.com
} 
ing for cnc-AlN thin films with (002) orientation and uniform textured on large silicon wafers for FBAR with effective piezoelectric performances. The characteristic of the cnc-AlN thin films were measured using X-ray diffraction (XRD), field emission scanning electron microscope (FESEM), transmission electron microscope (TEM), atomic force microscopy (AFM) and spectrum.

\section{Experimental}

RF reactive magnetron sputtering of an $\mathrm{Al}$ (99.999\% purity) target in a $\mathrm{N}_{2}$ (99.999\% purity) and $\mathrm{Ar}(99.999 \%$ purity) mixture atmosphere was used for deposition of cnc-AlN thin films. The distance target-to-substrate is about $70 \mathrm{~mm}$ and target diameter is $80 \mathrm{~mm}$. All samples were deposited on p-type Si $\left(\begin{array}{lll}1 & 0 & 0\end{array}\right)$ substrates of 3 inch with $1-10 \Omega \cdot \mathrm{cm}$ resistivity. Some controllable deposition parameters in the cnc-AlN films deposition experiments are shown in Table 1.

Table 1 The deposition parameters of cnc-AlN films

\begin{tabular}{ll}
\hline Deposition parameters & Value \\
Base pressure $(\mathrm{Pa})$ & $5 \times 10^{-5}$ \\
$\mathrm{RF}$ power $(\mathrm{W})$ & $100 \sim 250$ \\
$\mathrm{~N}_{2}$ flow rate $(\mathrm{sccm})$ & $0 \sim 20$ \\
Ar flow rate $(\mathrm{sccm})$ & $0 \sim 20$ \\
Sputtering pressure $(\mathrm{Pa})$ & $0.5 \sim 2.0$ \\
Substrate temperature $\left({ }^{\circ} \mathrm{C}\right)$ & $\mathrm{RT} \sim 300$ \\
\hline
\end{tabular}

The crystalline structure of the cnc-AlN films was investigated by XRD using a $\mathrm{Cu}-\mathrm{K} \alpha$ radiation $(\lambda=1.54187 \AA)$. The surface morphology of the cnc-AlN films was examined using FE-SEM. The crystalline grain size of the cnc-AlN films was observed by TEM. The surface roughness of the cnc-AlN films was determined using AFM. The optical transmission of the film was measured by UV spectrophotometer. The residual stress and defect density of the cnc-AlN films were also evaluated by Ramon spectroscopy.

\section{Results and discussion}

\section{The effects of deposition parameters on cnc-AlN films}

The deposition parameters, including RF power, pressure, ratio of $\mathrm{N}_{2} / \mathrm{Ar}$, and substrate temperature, have been investigated systematically and demonstrated significant effects on the crystalline structure and properties of AlN films. Figure 1 shows the XRD results of the AlN films deposited on silicon substrates with different RF power with $\mathrm{N}_{2} / \mathrm{Ar}$ of $70 \%$, substrate temperature of $300^{\circ} \mathrm{C}$, pressure of $1 \mathrm{~Pa}$. When the power was $100 \mathrm{~W}$, there are obvious non-crystalline structure feature as a broad diffraction peak in the XRD curve. With the increase of power, the AlN structure feature are becoming crystalline obvious and the AlN (002) and (100) diffraction peak becomes stronger and sharper. However, the AlN diffraction (100) peak becomes disappeared at the power of $200 \mathrm{~W}$ and a sharp AlN (002) peak remains.

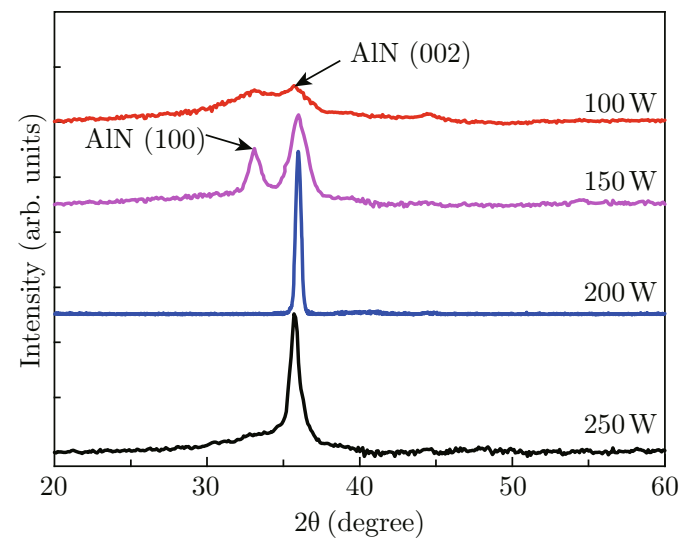

Fig. 1 The XRD results of the cnc-AlN films deposited on silicon substrates for different RF power levels.

The AlN (002) peak is observed at $35.94^{\circ}$ with a full width at half-maximum (FWHM) of $0.30^{\circ}$, which indicates that the preferential AlN growth orientation is along the wurtzite c-axis and perpendicular to the surface of substrates. According to the well-known Scherrer formula [6],

$$
D=\frac{0.94 \lambda}{\beta \cos \theta}
$$

where, $\mathrm{D}$ is the crystalline grain size, $\lambda$ is the wavelength of $\mathrm{Cu}-\mathrm{K} \alpha$ radiation, $\beta$ is the FWHM of diffraction peak, and $\theta$ is the position of diffraction peak. The crystalline grain size of AlN is estimated to be about $28 \mathrm{~nm}$ from the formula, which is similar to the value reported in the paper [7]. But, when the power is high to $250 \mathrm{~W}$, the $(002)$ peak becomes relatively broader, which may due to the high power induced increase of defects in the film nanocrystalline structure.

Figure 2 shows the XRD results of the cnc-AlN films deposited on the silicon substrates at different pressures with the RF power of $200 \mathrm{~W}, \mathrm{~N}_{2} / \mathrm{Ar}$ of $70 \%$, substrate temperature of $300^{\circ} \mathrm{C}$. It can be seen that as the pressure increased from $0.5 \mathrm{~Pa}$ to $2 \mathrm{~Pa}$ gradually, the FWHM of the AlN (002) diffraction peak decreases at first and then increases, and the optimum pressure is at $1 \mathrm{~Pa}$. This result may be explained that when the pressure was very low, the $\mathrm{Ar}^{+}$and $\mathrm{N}^{+}$ions with much high momentum were not suitable enough to form well crystallinenature. When the pressure was very high, the ratio of ion collision between each other became more frequent and weaker correspondingly [8], the energy of $\mathrm{Ar}^{+}$and $\mathrm{N}^{+}$ions may be not high enough for the formation of (002) crystal plane, which leads to texture decline in the film. 


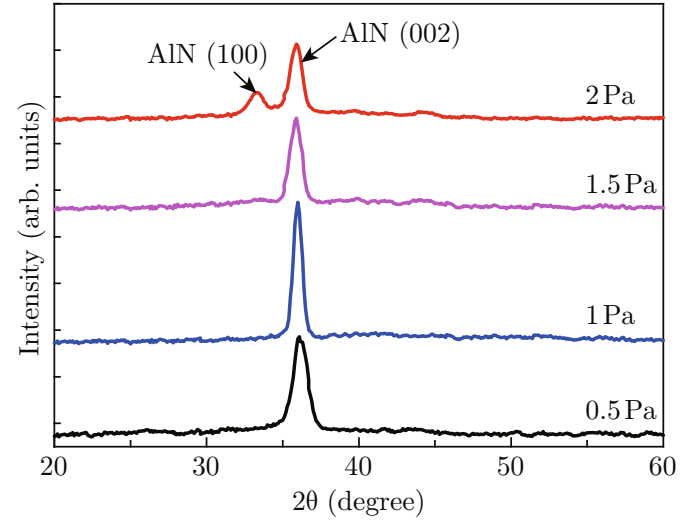

Fig. 2 The XRD results of the cnc-AlN films deposited on the silicon substrates for different pressure levels.

Figure 3 shows the XRD results of the cnc-AlN films deposited on the silicon substrates for different ratios of $\mathrm{N}_{2} / \mathrm{Ar}$ at the pressure of $1 \mathrm{~Pa}, \mathrm{RF}$ power of $200 \mathrm{~W}$, and substrate temperature of $300^{\circ} \mathrm{C}$. Only $\mathrm{Al}$ film was deposited when there was only $\mathrm{Ar}$ and without $\mathrm{N}_{2}$ for reaction in the sputtering process. With the increase of the $\mathrm{N}_{2}$ concentration, the cnc-AlN films were obtained and the AlN (002) diffraction peak becomes stronger and sharper. When the $\mathrm{N}_{2}$ was used only, the film deposition was very slow because of the lower the kinetic energy of $\mathrm{N}^{+}$ions inducing the decline of film quality.

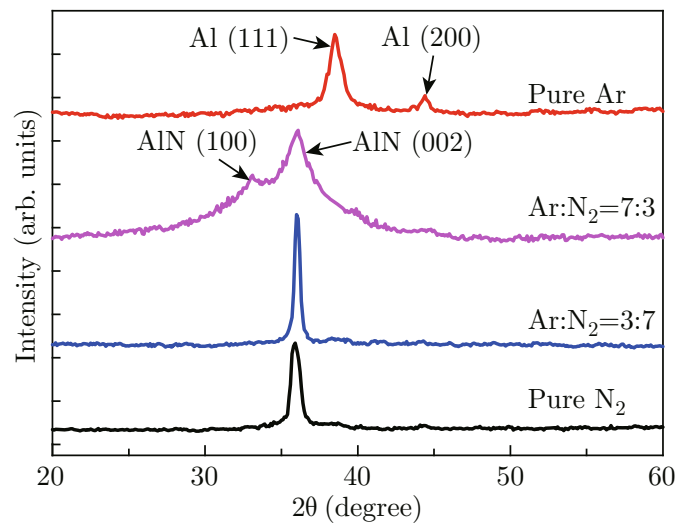

Fig. 3 The XRD results of the cnc-AlN films deposited on the silicon substrates for different ratios of $\mathrm{N}_{2}$ to Ar.

Figure 4 shows the XRD results of the cnc-AlN films deposited on the silicon substrates for different temperatures at the pressure of $1 \mathrm{~Pa}, \mathrm{RF}$ power of $200 \mathrm{~W}$, and $\mathrm{N}_{2} / \mathrm{Ar}$ of $70 \%$. It can be seen that the diffraction peak of AlN (002) and the FWHM decreases obviously with the temperature increase, which could attribute to the atoms diffusion ability increase on the growth surface and improves the crystalline quality of cnc-AlN films.

From the investigation above, one can see that good quality cnc-AlN films with (002) orientation can be deposited at an optimal condition around $200 \mathrm{~W}, 1.0 \mathrm{~Pa}$, $300^{\circ} \mathrm{C}$, and $\mathrm{N}_{2}$ concentration of $70 \%$, respectively.

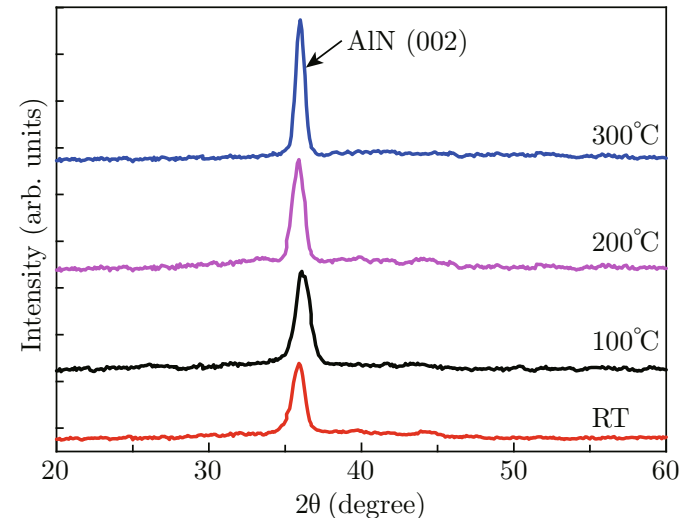

Fig. 4 The XRD results of the cnc-AlN films deposited on the silicon substrates for different temperature levels.

\section{The structure of (002) oriented cnc-AlN films}

The comprehensive features of cnc-AlN films, such as microstructure, grain size, and texture, have been observed intuitionally from TEM micrographs. TEM bright field and dark field images were used to examine the microstructure and grain size of the A1N film, respectively. While the selected area electron diffraction (SAED) was utilized to study the crystalline phase present. Figure 5(a) shows a cross-section bright-filed (BF) TEM micrograph, together with the SAED pattern in Fig. 5(b) and the dark-filed TEM micrograph in Fig. 5(c). The in-plane nanocrystalline grains are columnar with grain size of about $24 \mathrm{~nm}$, which is similar with the value calculated by Scherrer formula. The SAED pattern shown in Fig. 5(c) indicated a crystalline phase of AlN can be obtained. As for AlN, a preferred orientation is already seen from the arcing of the (002) diffraction spots around the growth axis, according to the occurring diffraction rings. In addition, according to the SAED pattern, it is obviously typical of a

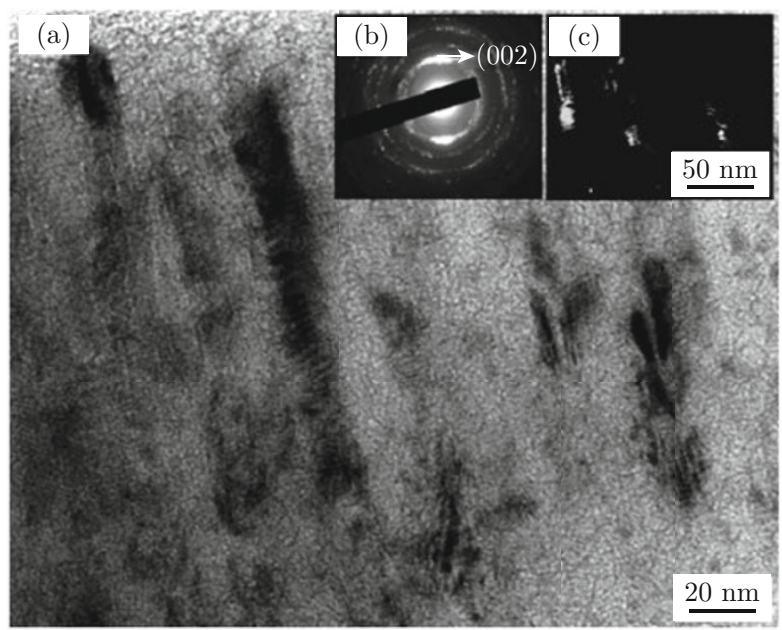

Fig. 5 The TEM micrographs obtained for cnc-AlN thin films: (a) the bright filed image; (b) the SAED pattern; (c) the dark filed image. 
polycrystalline film. Moreover, the diffraction spots are very intense, which proved that the film is well crystallite.

Figure 6(a) and (b) show SEM and AFM of the surface morphology of cnc-AlN films deposited on silicon substrates by using the optimal deposition parameters, and Fig. 6(c) shows the cross-sectional image. The cnc-AlN films demonstrate clearly a hummock-like surface morphology with smooth, homogeneous, uncracked, compact and dense. The surface morphology consists of a large number of crystalline grains with compact columnar structure. There are many grain boundaries among columns and the RMS was in an average of $1.29 \mathrm{~nm}$ which was measured by the low-high ruggedness in the AFM.
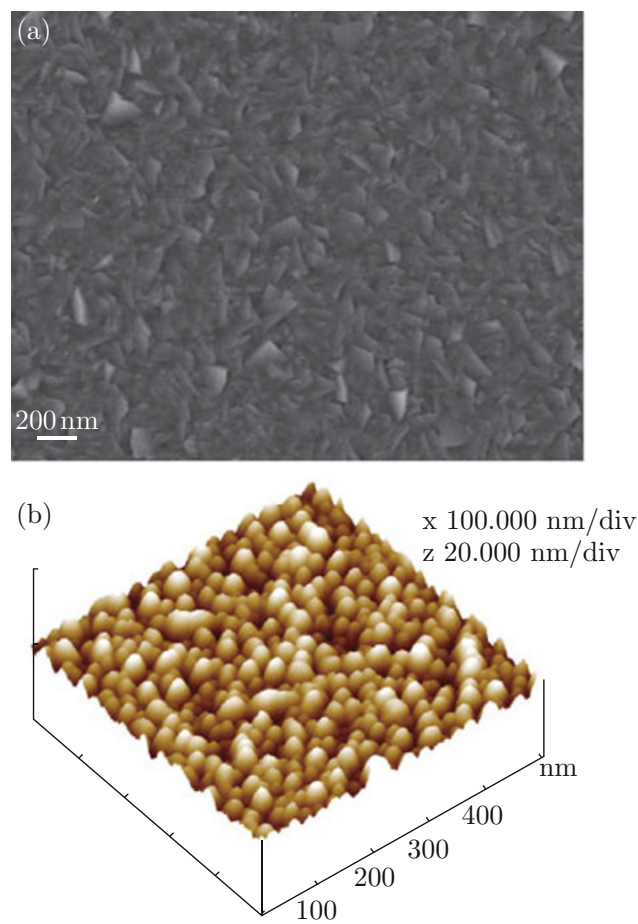

(c)

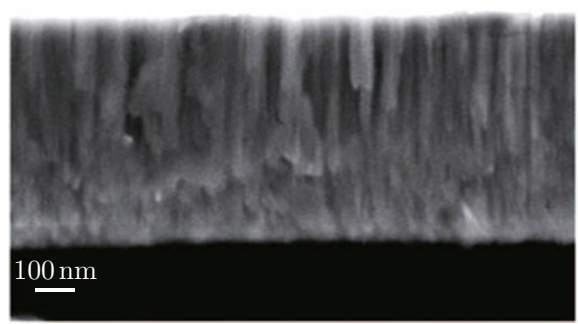

Fig. 6 The morphology of cnc-AlN thin films: (a) SEM image of the surface; (b) AFM image of the surface; (c) SEM image of the cross-section.

Figure 7(a) demonstrates the UV transmittance spectrum of cnc-AlN thin films with the thickness of $500 \mathrm{~nm}$ deposited on a glass substrate. The optical properties of the cnc-AlN films transparent in appearance with average transmittance of about $92.6 \%$ in the wavelength range of 200-800 $\mathrm{nm}$. The fundamental absorption edge for the cnc-AlN films is found to be near $290 \mathrm{~nm}$.
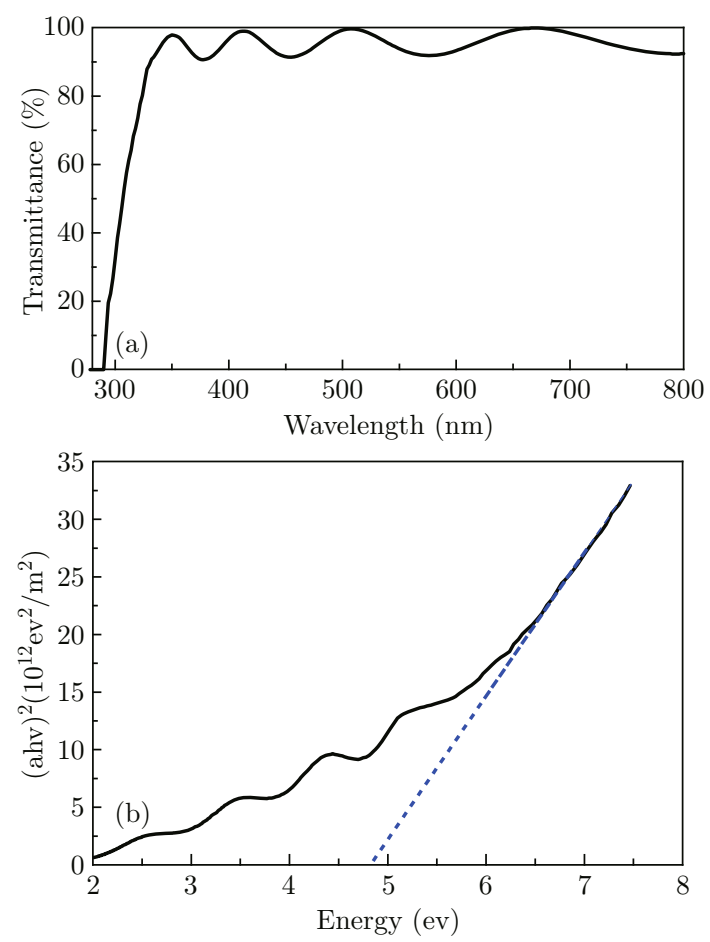

Fig. 7 (a) The UV spectrum of cnc-AlN films deposited on glass. (b) The inset shows the typical $(\alpha h \nu)^{2} \sim h \nu$ curve for the cnc-AlN films.

The optical band gap of the cnc-AlN films have been calculated with the Tauc equation [9]:

$$
(\alpha h v)^{2}=B\left(h v-E_{g}\right)
$$

$\alpha$ is the absorption coefficient, $B$ is a proportional constant related to the material, $h v$ is the photon energy of incident light and $E_{g}$ is the optical band gap, respectively. The optical band gap is obtained by extrapolating the tangential line to the photon energy axis in the plot of $(\alpha h v)^{2}$ as a function of hv. Figure 7(b) shows the typical $(\alpha h v)^{2} \sim h \nu$ curve for the cnc-AlN films. The optical band gap for the cnc-AlN films is estimated about $4.8 \mathrm{eV}$, which is similar to the reported data [10].

Figure 8 shows a typical Raman spectrum of the cncAlN films deposited on silicon substrates. Two obvious intense peaks can be observed from the spectrum, one at $519 \mathrm{~cm}^{-1}$ originates from the silicon substrate, another at $660 \mathrm{~cm}^{-1}$ corresponds to the $\mathrm{E}_{2}$ (high) mode of AlN, of which the frequency is higher than the standard frequency of $\mathrm{E}_{2}$ at $657.4 \mathrm{~cm}^{-1}$ [11]. This reveals there are small residual compressive stresses in the cnc-AlN films, which is possibly induced by the difference of the thermal expansion coefficient between the film and the Si substrate. 


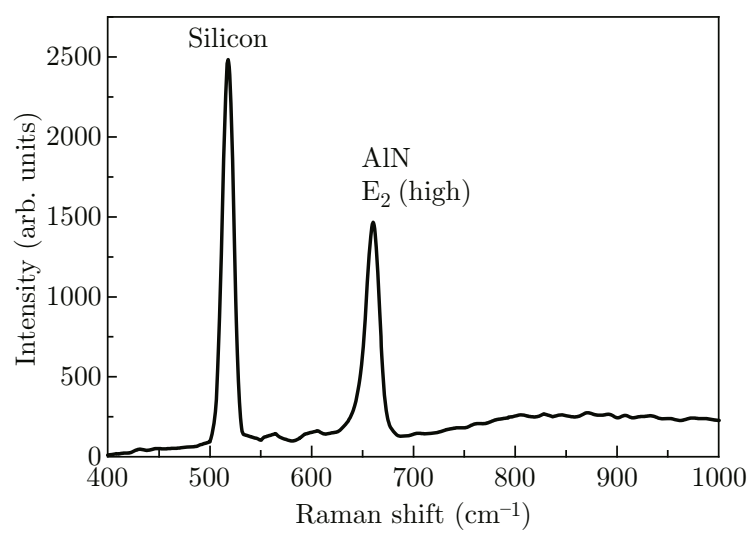

Fig. 8 The typical Raman spectrum of the cnc-AlN films deposited on silicon substrates.

The (002) textured cnc-AlN films have been used crucial for film body acoustic rasonator (FBAR) as the piezoelectric film which is most crucial in quality. Figure 9 shows a schematic of a FBAR sample and the measured frequency response curve. The center frequency of the FBAR is about $2.2 \mathrm{GHz}$ and the insertion loss is about $30 \mathrm{~dB}$. Results showed the devices exhibited excellent performance with the quality factor (Q) about 385 and the effective electromechanical coupling coefficient $\left(\mathrm{k}_{\text {eff }}^{2}\right)$ about $3.4 \%[12,13]$.
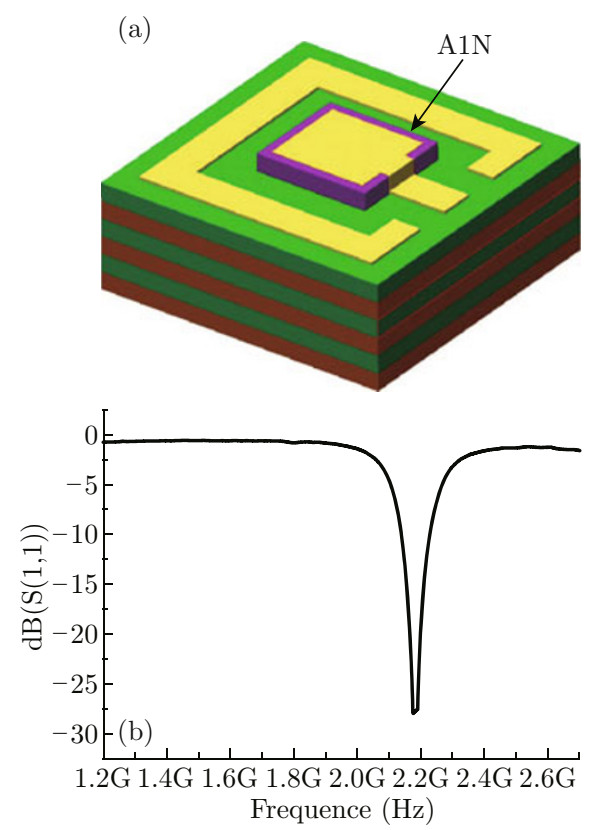

Fig. 9 The schematic of FBAR (a) schematic of structure; (b) frequency response curve.

\section{Conclusions}

Well (002) oriented cnc-AlN films have been deposited on silicon substrates by $\mathrm{RF}$ reactive magnetron sputtering with texture. The effect of process param- eters on properties of cnc-AlN films has been demonstrated systematically. A hummock-like surface morphology with smooth, homogeneous, uncracked, compact and dense, have been observed by SEM and the crystallite size in the cnc-AlN films is about $24 \mathrm{~nm}$ which was measured by TEM observation. The average transmittance is about $92.6 \%$ and the optical band gap is estimated to be about $4.8 \mathrm{eV}$. An intense peak can be observed at $660 \mathrm{~cm}^{-1}$ from the Raman spectrum, which proved the residual compressive stress in the cnc-AlN films. The high quality of the cnc-AlN films have been proved with good performance as a piezoelectric film in the critical structure of film bulk acoustic resonator.

\section{References}

[1] A. Khanna and D. G. Bhat, J. Vac. Sci. Technol. A 25, 557 (2007). http://dx.doi.org/10.1116/1.2730513

[2] M. Clement, E. Iborra, J. Sangrador, A. Sanz-Hervas, L. Vergara and M. Aguilar, J. Appl. Phys. 94, 1495 (2003). http://dx.doi.org/10.1063/1.1587267

[3] T. P. Drusedau and J. Blasing, Thin Solid Films 377, 27 (2000). http://dx.doi.org/10.1016/ S0040-6090(00) 01380-8

[4] M. Clement, E. Iborra, J. Sangrador, et al., J. Appl. Phys. 94, 1495 (2003). http://dx.doi.org/10.1063/ 1.1587267

[5] X. H. Xu, H. S. Wu, C. J. Zhang, et al., Thin Solid Films 388, 62 (2001). http://dx.doi.org/10.1016/ S0040-6090 (00) 01914-3

[6] S. S. Lin, J. L. Huang and P. Sajgalik, Surf. Coatings Technol. 190, 39 (2005). http://dx.doi.org/10. $1016 / j$. surf coat. 2004.03.022

[7] S. Chon, J. Cryst. Growth 326, 179 (2011). http:// dx.doi.org/10.1016/j.jcrysgro. 2011.01.092

[8] E. Valcheva, S. Dimitrov, D. Manova, et al., Surf. Coat. Tech. 202, 2319 (2008). http://dx.doi.org/10. 1016/j . surf coat. 2007.08.051

[9] F. Yakuphanoglu, M. Sekerci and O. F. Ozturk, Opt. Commun. 239, 275 (2004). http://dx.doi.org/10. 1016/j.optcom. 2004.05.038

[10] B. Subramanian, V. Swaminathan and M. Jayachandran, Current Appl. Phys. 11, 43 (2011). http://dx. doi.org/10.1016/j.cap. 2010.06.016

[11] T. Prokofyeva, M. Seon, J. Vanbuskirk, M. Holtz, S. A. Nikishin, N. N.Faleev, H. Temkin, and S. Zollner, Phys. Rev. B 63, 125313 (2001). http://dx.doi.org/ 10.1103/PhysRevB.63.125313

[12] D. T. Phan, G. S. Chung, Appl. Surf. Sci. 257, 8696 (2011). http://dx.doi.org/10.1016/j.apsusc. 2011.05 .050

[13] Q. X. Su, P. Kirby, E. Komuro, M. Imura, Q. Zhang and R. Whatmore, IEEE Trans. Microwave Theory Tech 49, 769 (2001). http://dx.doi.org/10.1109/ 22.915462 